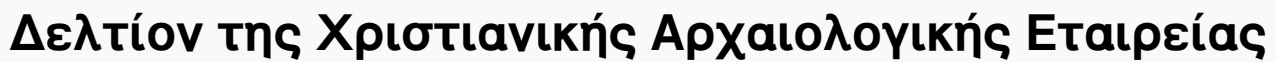

Tó 18 (1995)

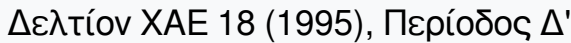

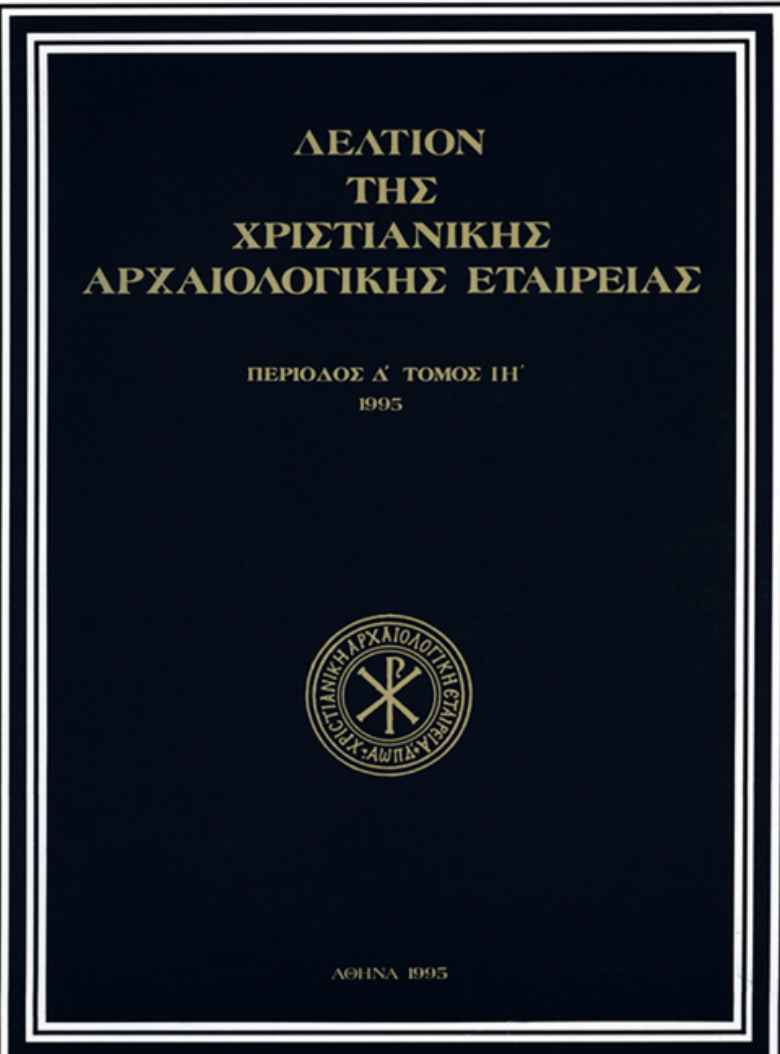

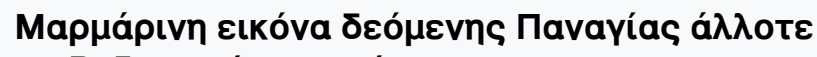

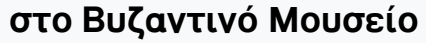

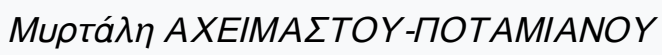

doi: $10.12681 /$ dchae. 1138

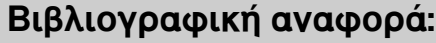

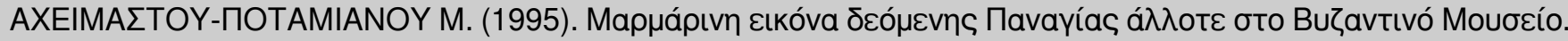

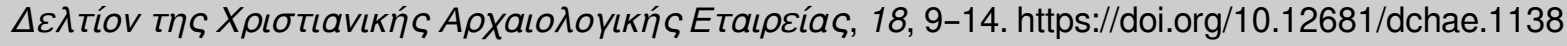




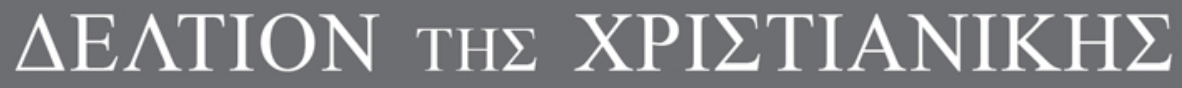 APXAIO $\Lambda$ OГIKH $\Sigma$ ETAIPEIA $\Sigma$}

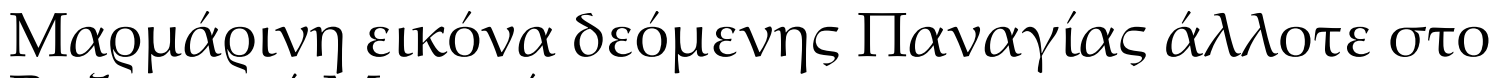

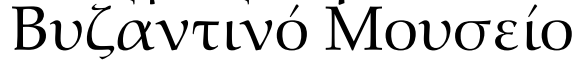

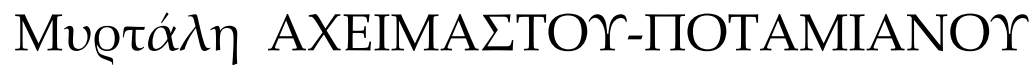

$\Delta \varepsilon \lambda \tau$ tíov XAE 18 (1995), Пв@íodos $\Delta^{\prime} \bullet \Sigma \varepsilon \lambda .9-14$ A@HNA 1995 


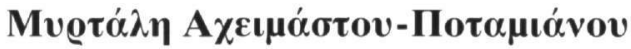

\section{MAPMAPINH EIKONA $\triangle$ EOMENH $\Sigma$ ПANAГIA $\Sigma$ A $\Lambda$ ATE $\Sigma$ TO BYZANTINO MOY $\Sigma E I O$}

$\mathrm{H}$

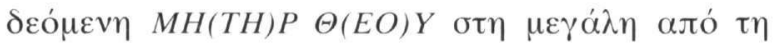

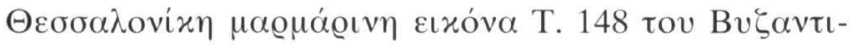

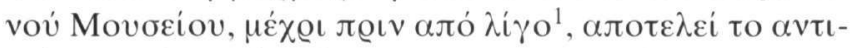

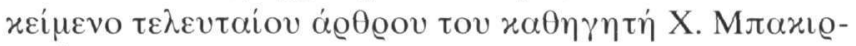

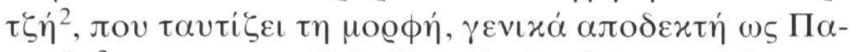

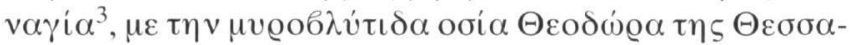

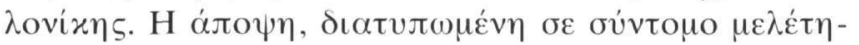

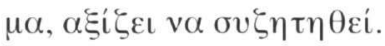

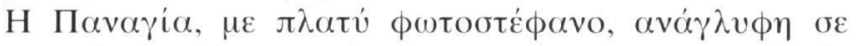

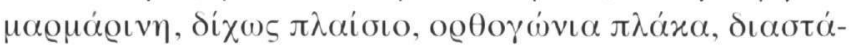

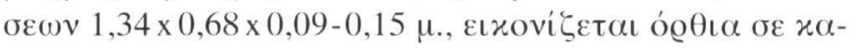

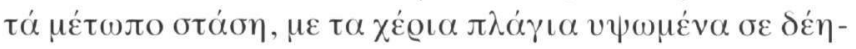

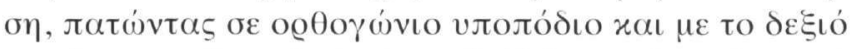

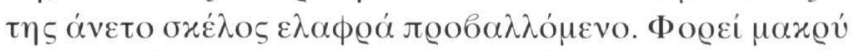

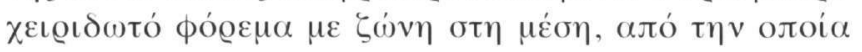

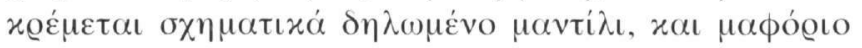

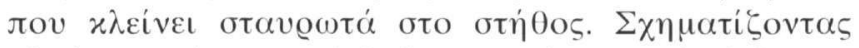

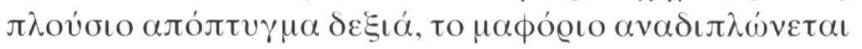

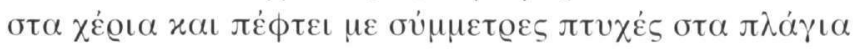

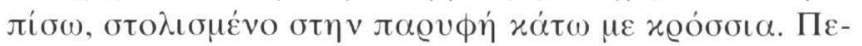

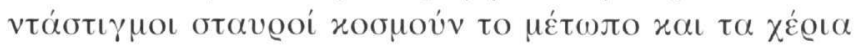

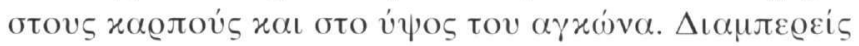

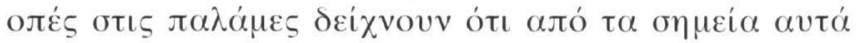

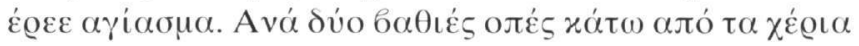

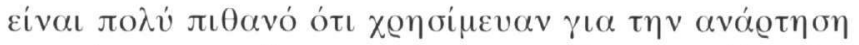

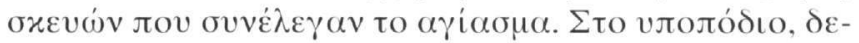

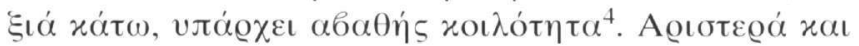

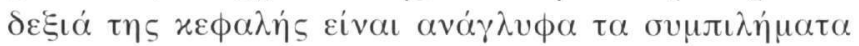

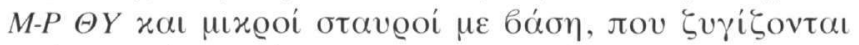

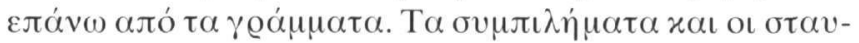

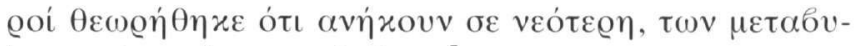

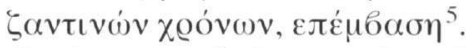

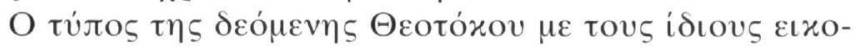

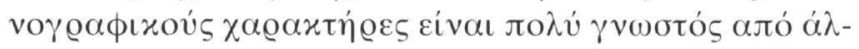

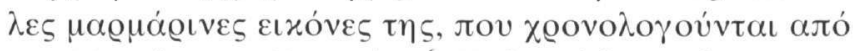

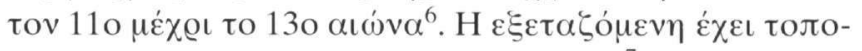

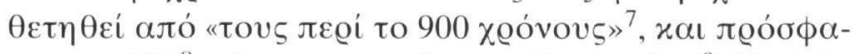

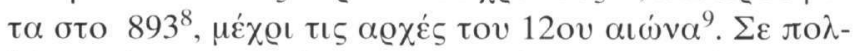

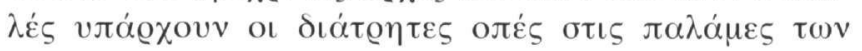

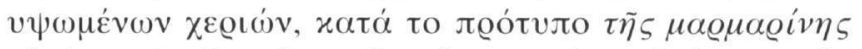

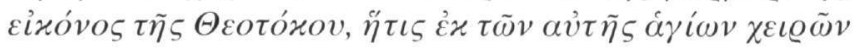

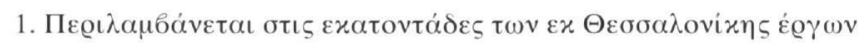

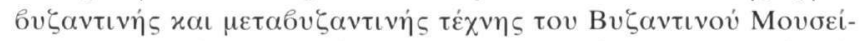

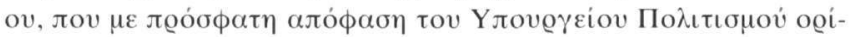

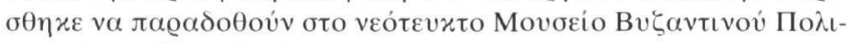

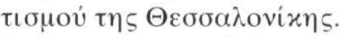

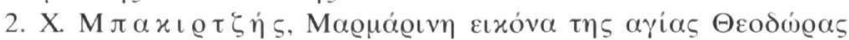

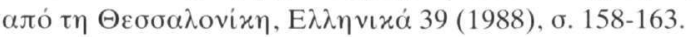

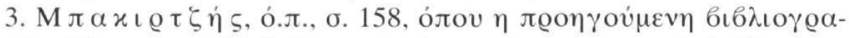

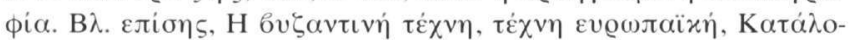

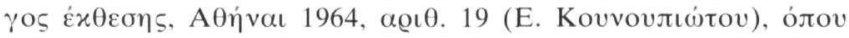

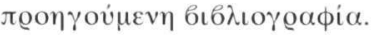

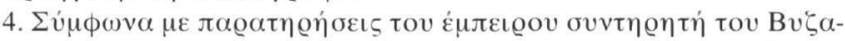

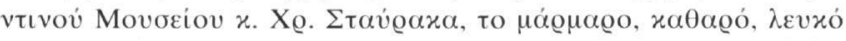

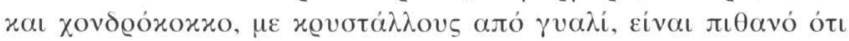

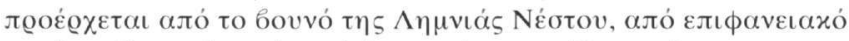

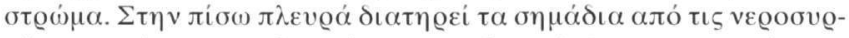

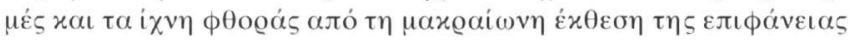

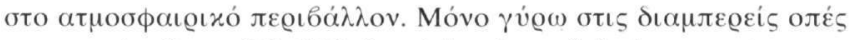

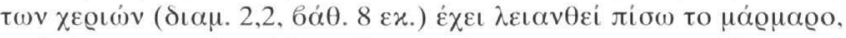

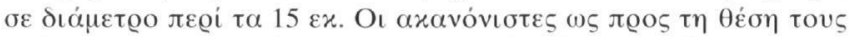

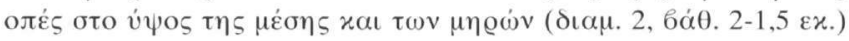

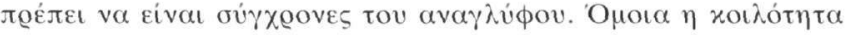

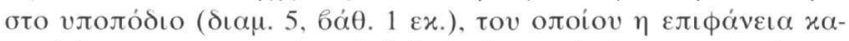

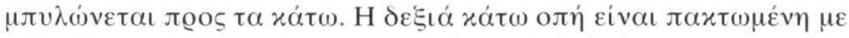

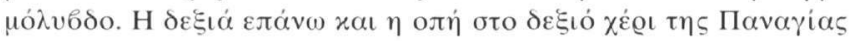

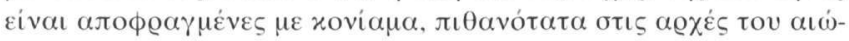

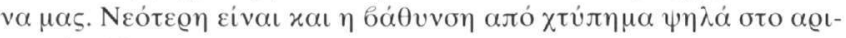

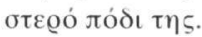

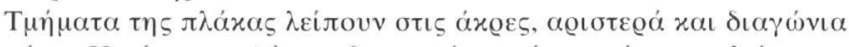

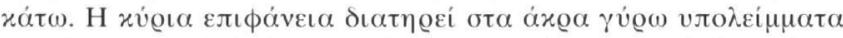

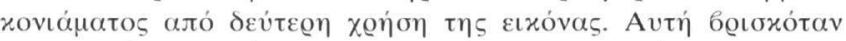

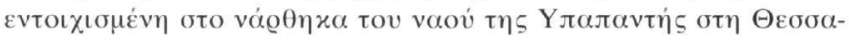

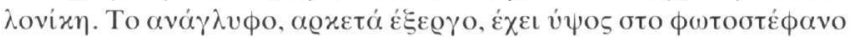

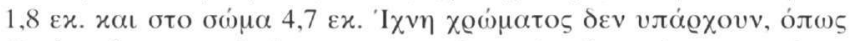

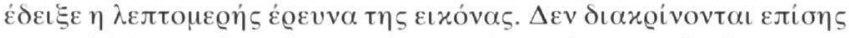

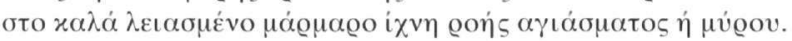

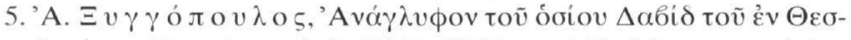

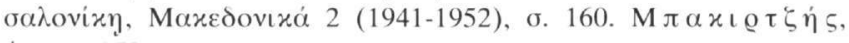
ó.л., б. 159.

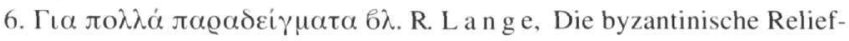

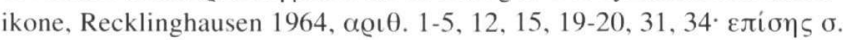
22, riv. V.

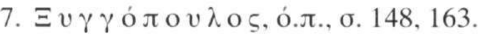

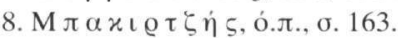

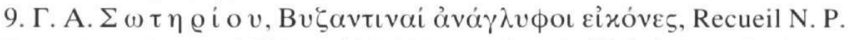

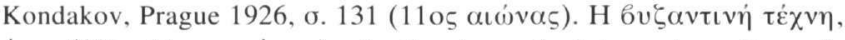

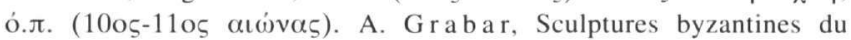

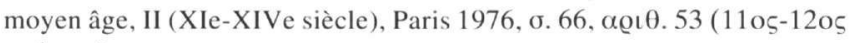

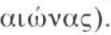




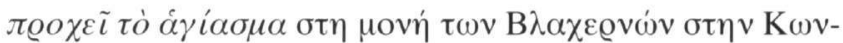

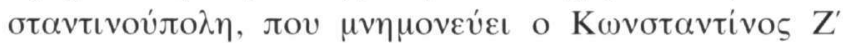

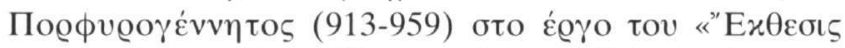

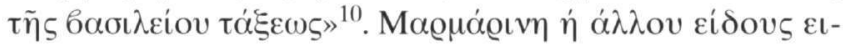

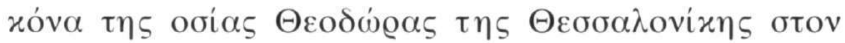

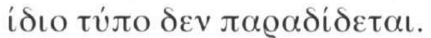

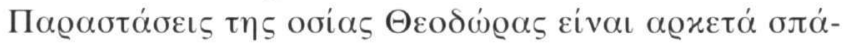

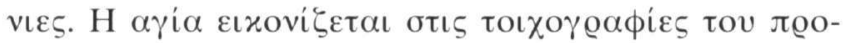

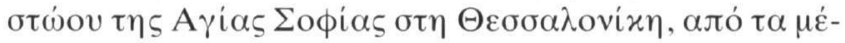

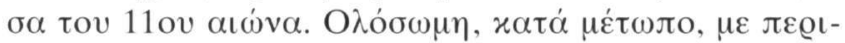

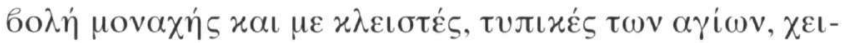

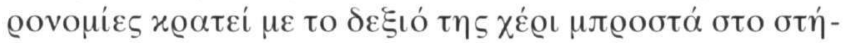

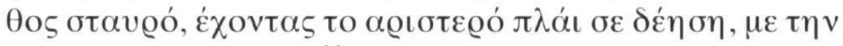

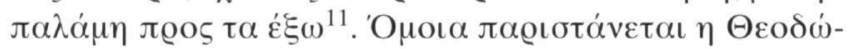

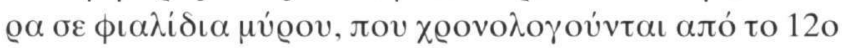

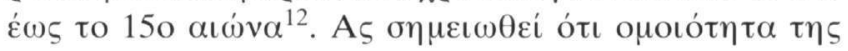

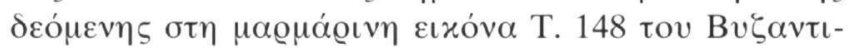

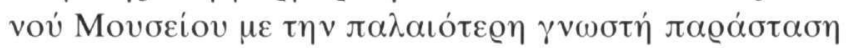

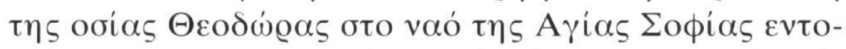

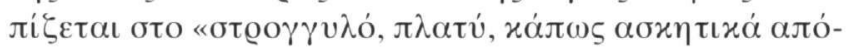

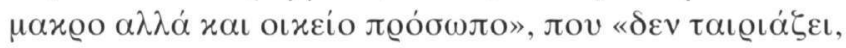

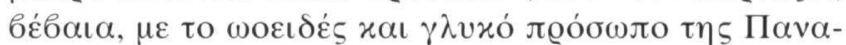

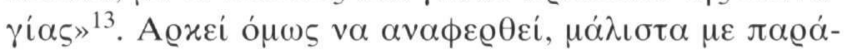

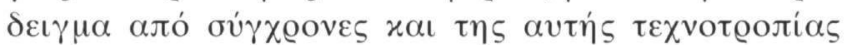

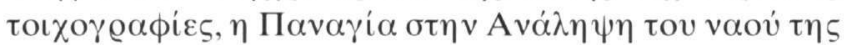

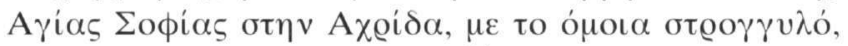

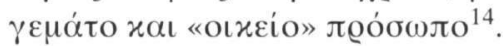

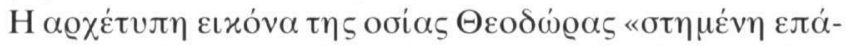

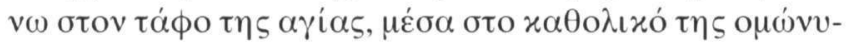

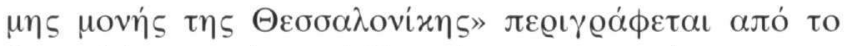

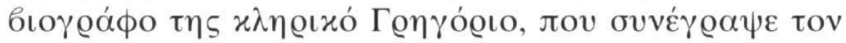

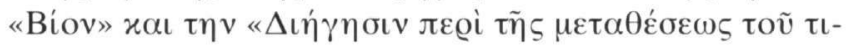

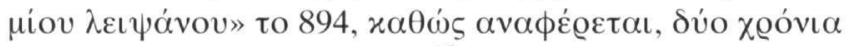

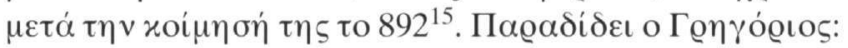

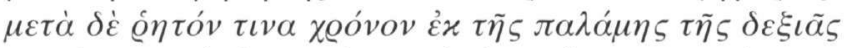

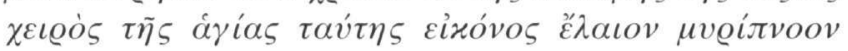

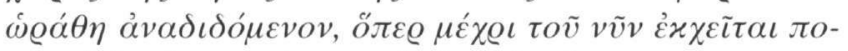
$\tau \alpha \mu \eta \delta o ́ v, \omega \varsigma \varsigma$ «

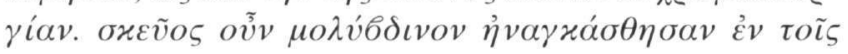

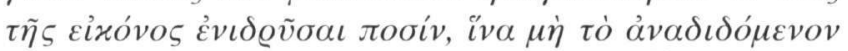

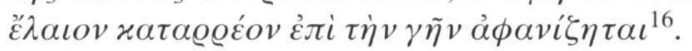

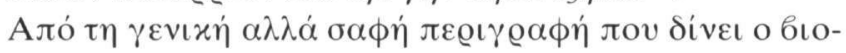

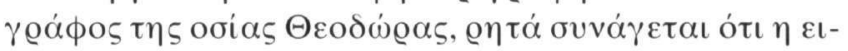

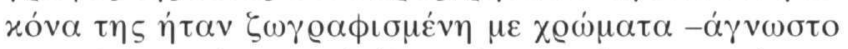

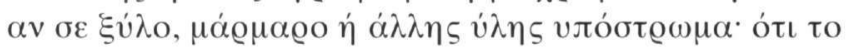

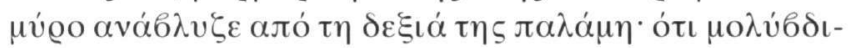

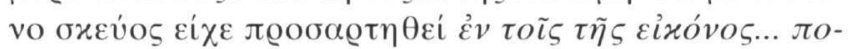

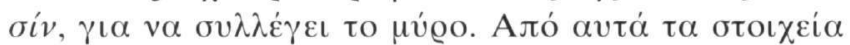

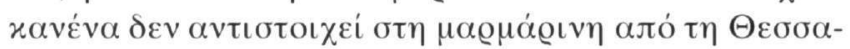

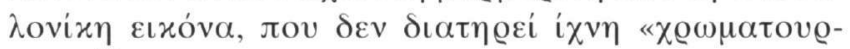

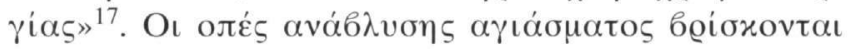

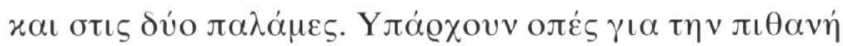

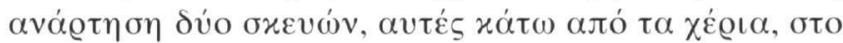

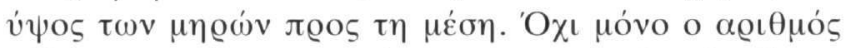

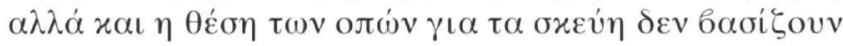
$\tau \eta v \varepsilon \pi \mathrm{r} \theta v \mu \eta \tau \dot{~ o \mu o t o ́ t \eta \tau \alpha ~ \mu \varepsilon ~ \tau \eta v ~ \alpha v \alpha \phi \varepsilon @ o ́ \mu \varepsilon v \eta ~ \alpha \pi o ́ ~}$

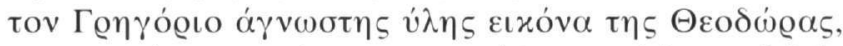

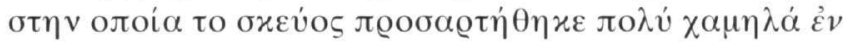

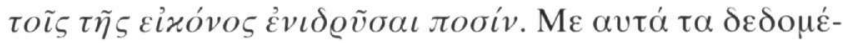

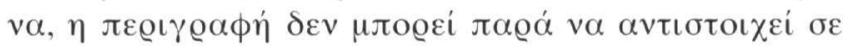

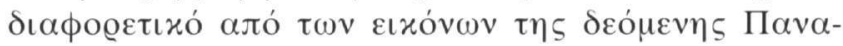

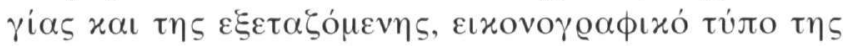

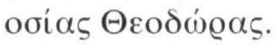

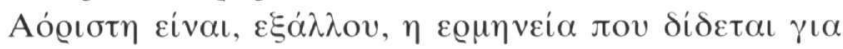

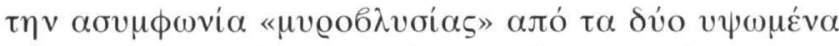

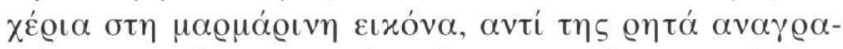

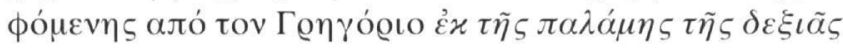

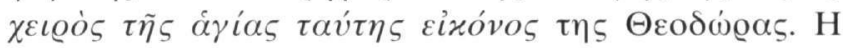

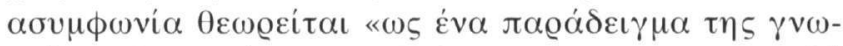

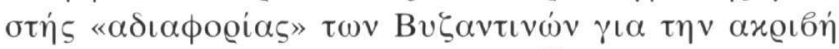

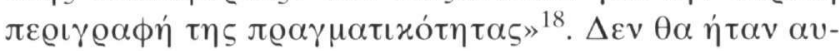

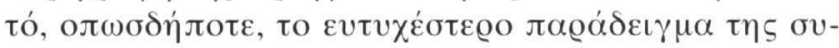

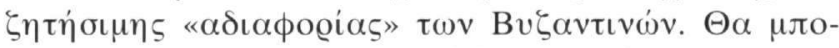

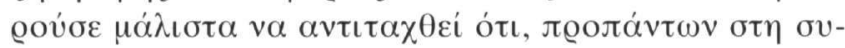

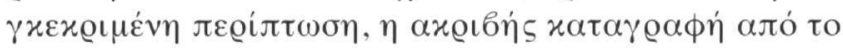

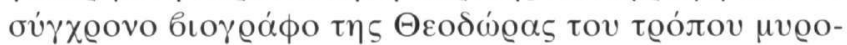

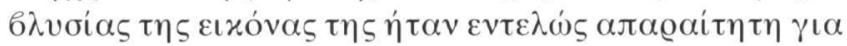

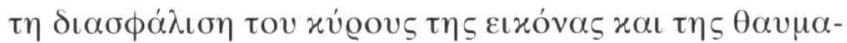

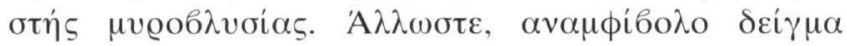

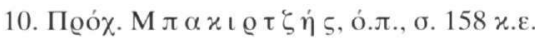

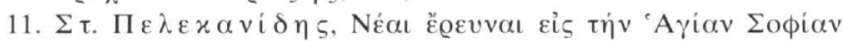

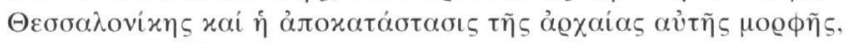

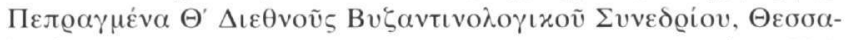

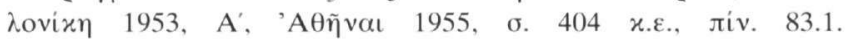

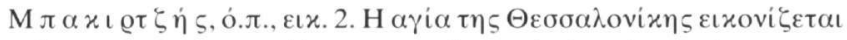

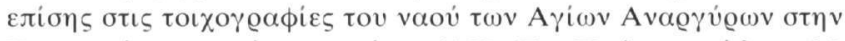

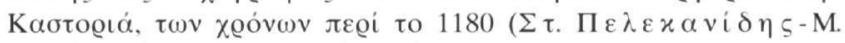
$\mathrm{X} \alpha \tau \zeta \eta \delta \dot{\alpha} x \eta \varsigma, \mathrm{K} \alpha \sigma \tau o \varrho \iota \dot{,}, \mathrm{A} \theta \dot{v} v \alpha$ 1984, б. 43).

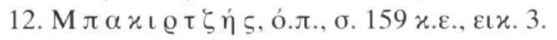

13. 'О.л., б. 159.

14. R. Hamann-Mac Lean $x \alpha \iota$ H. Hallensleben, Die Monumentalmalerei in Serbien und Makedonien, vom 11. bis zum frühen 14. Jahrhundert, Giessen 1963, عıx. 15.

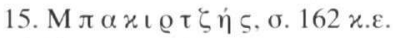

16. 'О.л., б. 162.

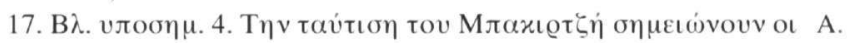
Kazhdan $x \alpha$ H. Maguire (Byzantine Hagiographical Texts as

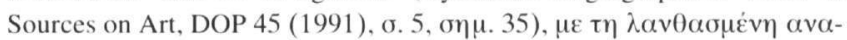

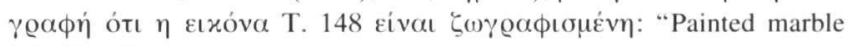
relief icon".

18. $\mathrm{M} \pi \alpha x \iota \mathrm{Q} \tau \dot{\eta} \varsigma, \dot{.} . \pi ., 163$. 


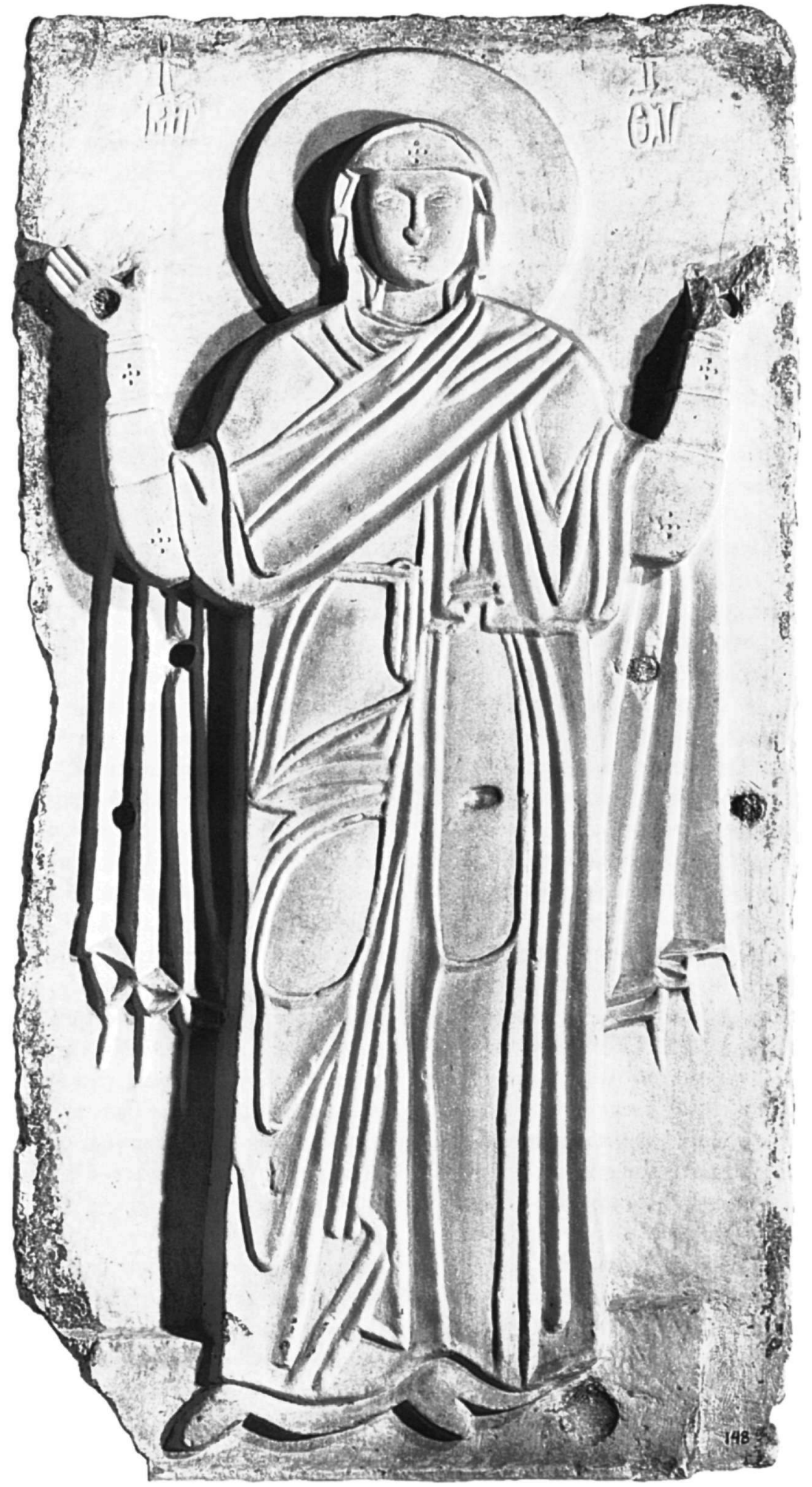

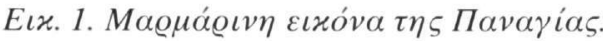




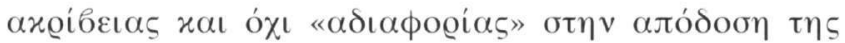

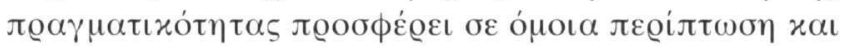

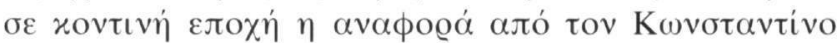

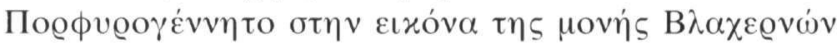

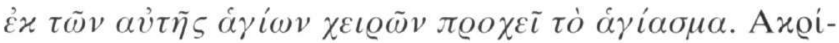

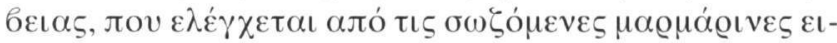

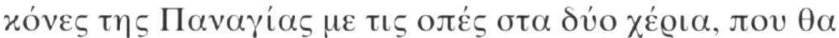

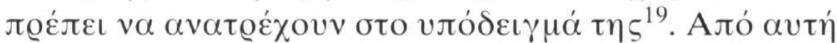

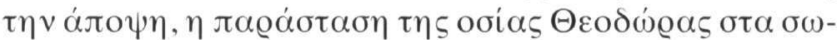

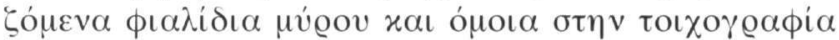

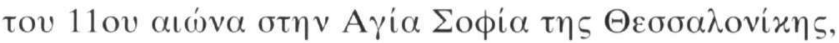

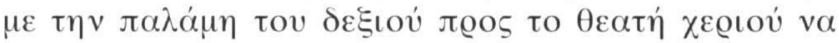

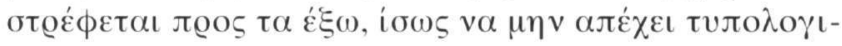

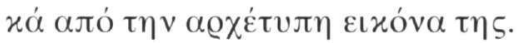

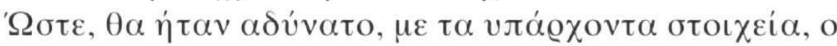

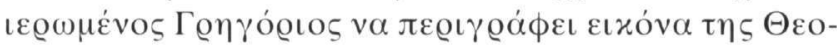

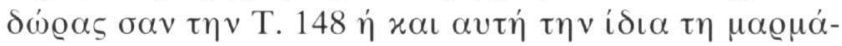

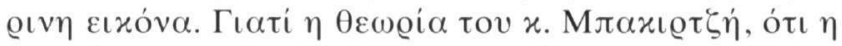

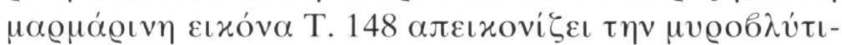

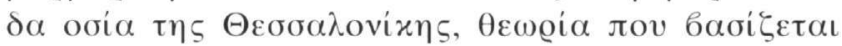

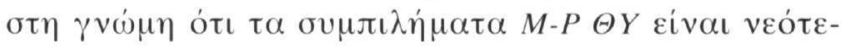

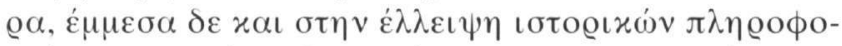

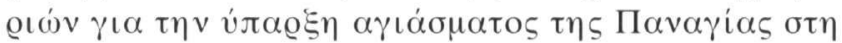

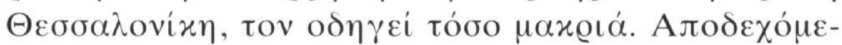

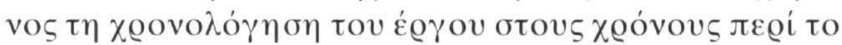

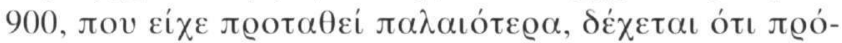

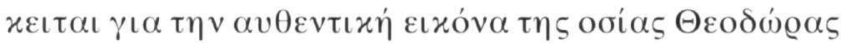

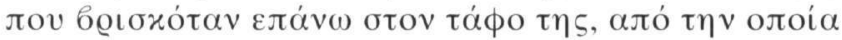

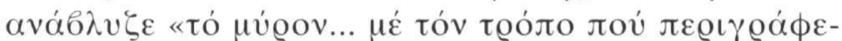

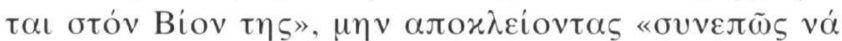

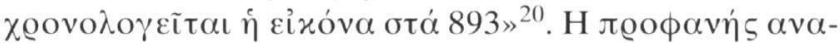

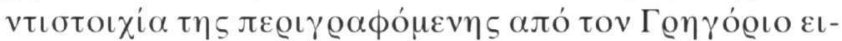

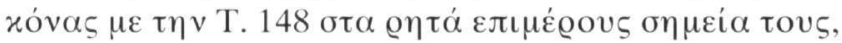

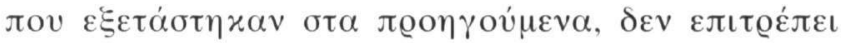

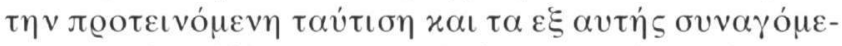

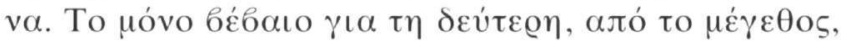

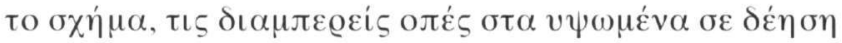

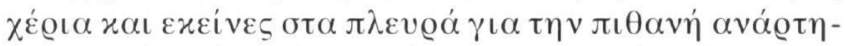

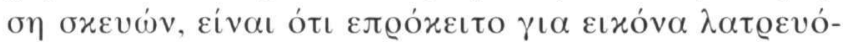

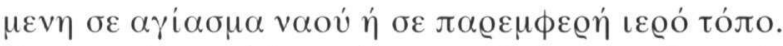

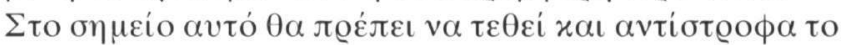

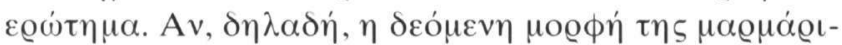

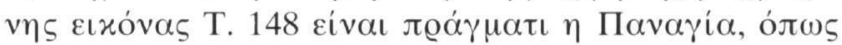

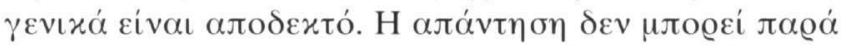

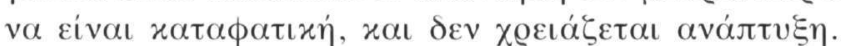

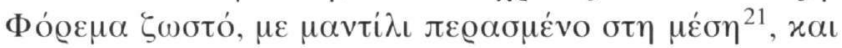

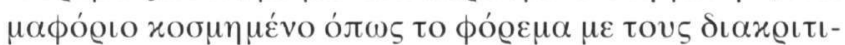

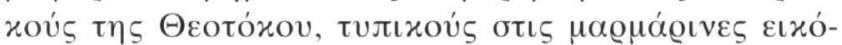

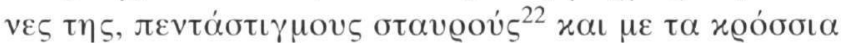

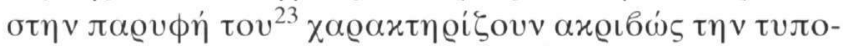

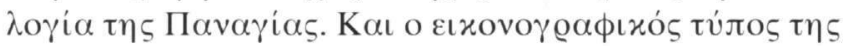

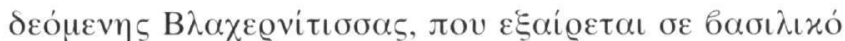

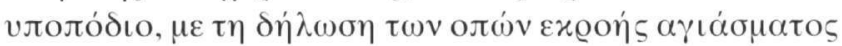

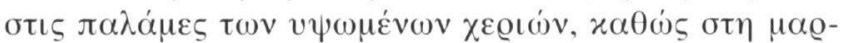

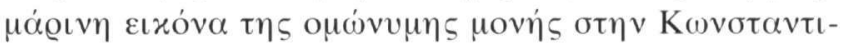

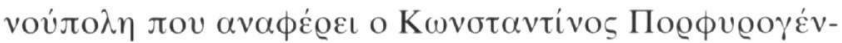

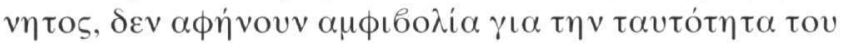

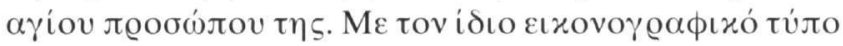

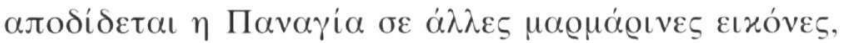

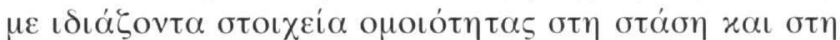

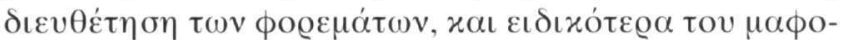

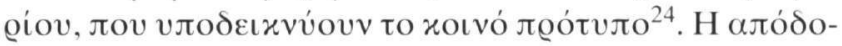

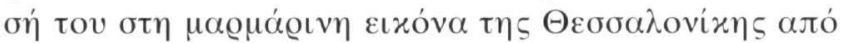

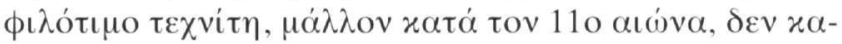

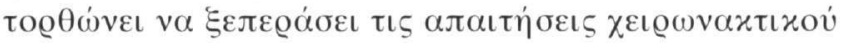

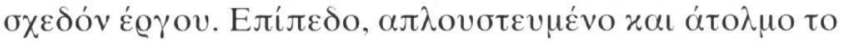
$\alpha v \alpha ́ \gamma \lambda v \phi o, \varepsilon \pi \iota \mu \varepsilon \lambda \omega ́ \varsigma \lambda \varepsilon \iota \alpha \sigma \mu \varepsilon \dot{v o,} \pi 0 v \theta \varepsilon v \alpha \dot{\delta} \delta \varepsilon v \pi \varrho o \delta i \delta \varepsilon \iota$

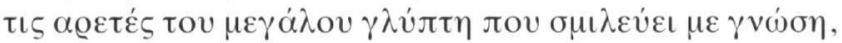

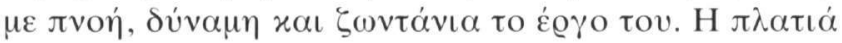

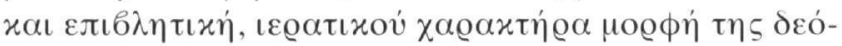

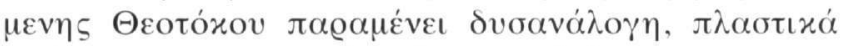

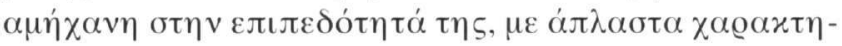

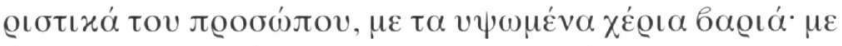

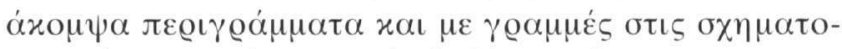

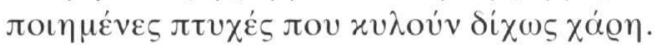

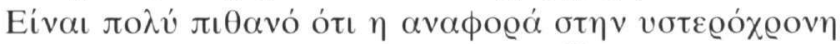

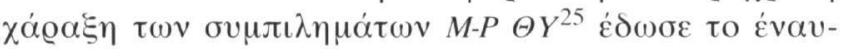

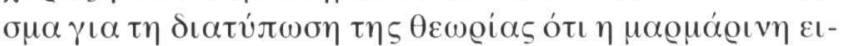

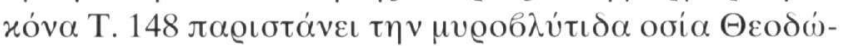

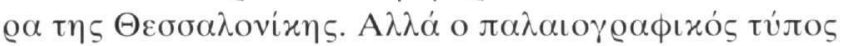

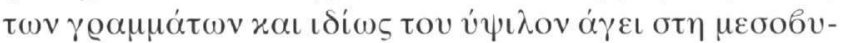

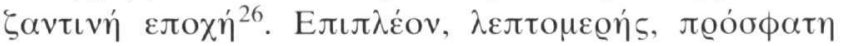

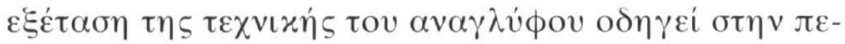

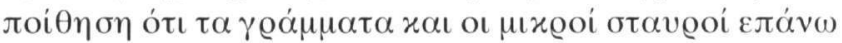

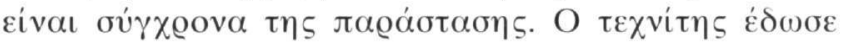

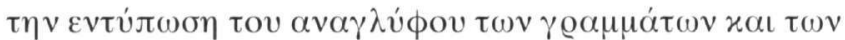

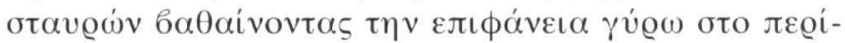

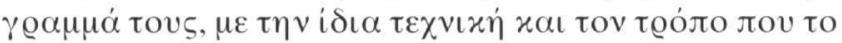

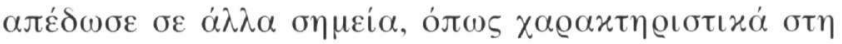

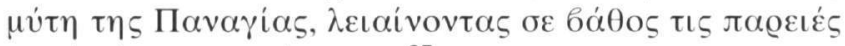

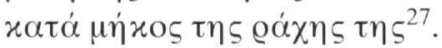

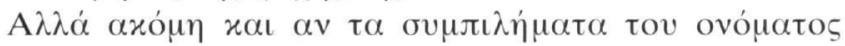

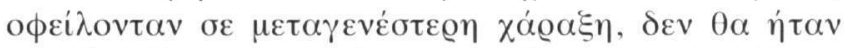

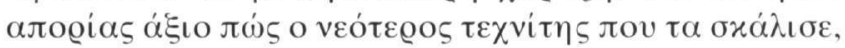

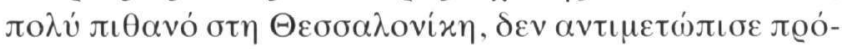

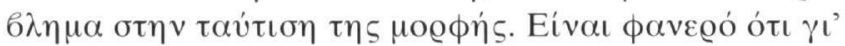

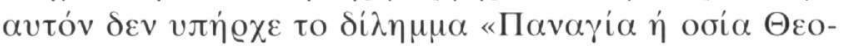

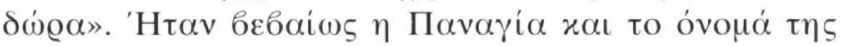

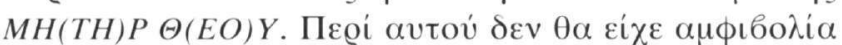

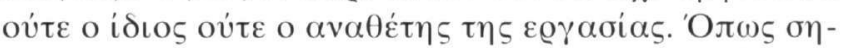

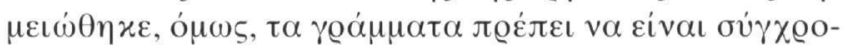

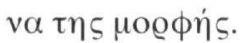




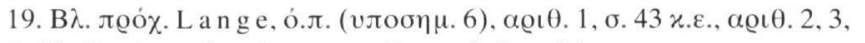

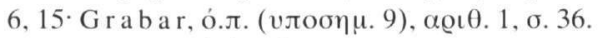

20. $\mathrm{M} \pi \alpha x \iota \mathrm{\tau} \zeta \dot{\eta} \varsigma$, ó. $\pi$.

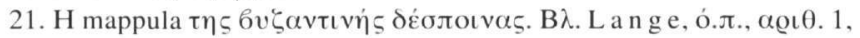

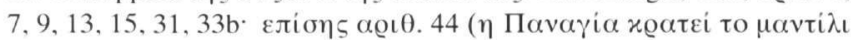

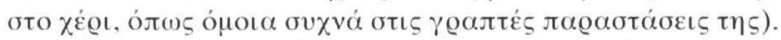

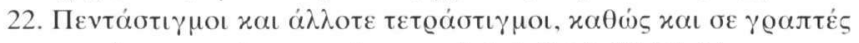

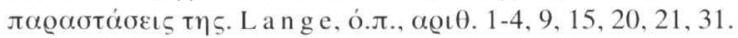

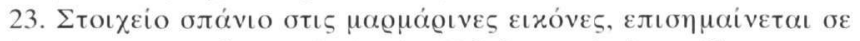

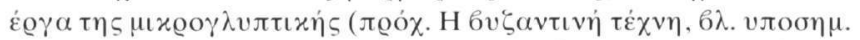

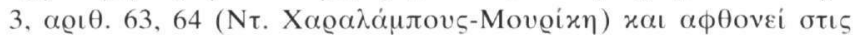

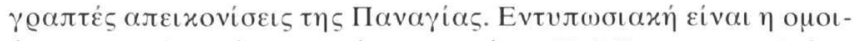

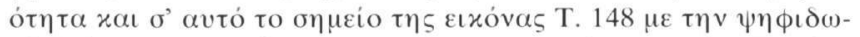

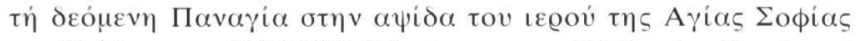
tov Kıย́bov, tov 1043-1046 ( V . L a z a re v, Old Russian Murals and

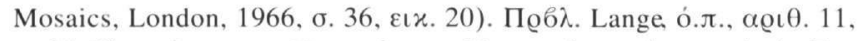

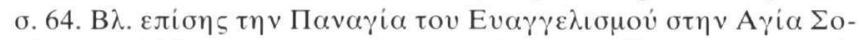

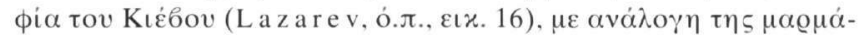

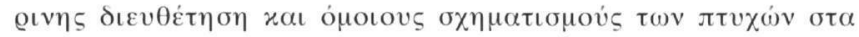
$\phi о \varrho \varepsilon ́ \mu \alpha \tau \alpha$.

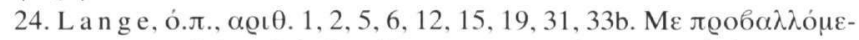

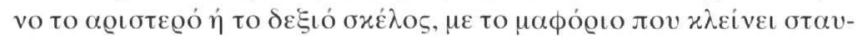

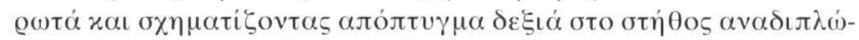

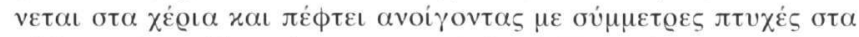

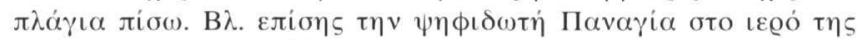

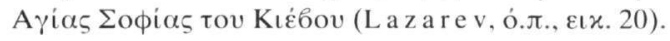

25. В $\lambda$. ขлобпц. 5.

26. $6 \lambda$. Lan g e, о́.л., $\alpha \varrho \iota \theta .9$ xa 10.

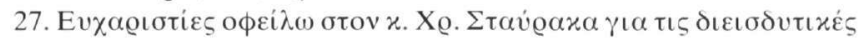
$\pi \alpha \varrho \alpha \tau \eta \emptyset \dot{\sigma \varepsilon} ı \varsigma$ тov. 


\title{
Myrtali Acheimastou-Potamianou
}

\author{
MARBLE ICON OF THE VIRGIN ORANT \\ ONCE IN THE BYZANTINE MUSEUM
}

$\mathrm{T}$ he Orant $\mathrm{MH}(\mathrm{TH}) \mathrm{P} \Theta(\mathrm{EO}) \mathrm{Y}$ in the large marble icon from Thessaloniki, T. 148 of the Byzantine Museum and now in the Museum of Byzantine Civilisation in Thessaloniki, was identified in a recent study with the myrrhproducing depiction of Hosia Theodora ( $\mathrm{C} \mathrm{h} . \mathrm{B}$ a k i r $\mathrm{z}$ i s, Marble icon of St. Theodora from Thessaloniki [in Greek], Ellinika 39 (1988), 158-63). Indeed, it is suggested that this is the icon mentioned by Theodora's biographer, Gregory, as being at her tomb in Thessaloniki (d. 892), and thus a possible date of 893 is given. It is also suggested, based in part on an earlier theory, that the incisions M-P $\Theta Y$ (Mother of God) were incised in post-Byzantine times and that the icon can thus be dated to around 900 . Gregory informs us that the icon at the tomb of Theodora was painted; the myrrh flowed "like a river" from the palm of her right hand and washed away the colours, necessitating the placement of a lead vessel at the icon's base. None of these clearly stated features are compatible with the marble icon T. 148, which preserves no trace of paint. Holes have been opened through the palms of the raised hands of the Virgin, and two on each arm further down, towards the waist, for the possible attachment of a vessel made of lead or some other material. The disparity between the written and archaeological evidence is attributed to the "disinterest" of the Byzantines when it came to providing accurate descriptions, in this case to the biographer of Theodora. The exact description, however, provided by Gregory, a contemporary of the Saint, of the manner in which the myrrh was produced would have been absolutely necessary in order to establish the authority of the icon and the miraculous production of myrrh. An undoubted example of exactitude in a similar circumstance, closely related chronologically, comes from the description by Constantine VII Porphyrogennitos of the marble icon of the Virgin in the Blachernae monastery in Constantinopole, "from whose holy hands the hagiasma pours forth". This exactitude is controlled by the extant marble icons of the Virgin Orant with the holes in both hands, which must be considered as having had the Blachernae icon as their model, such as T. 148.

Depictions of Hosia Theodora orant are not preserved. In her earliest known depiction, in a wall-painting in the church of Hagia Sophia in Thessaloniki from the middle of the 11th century, and in those on the 12th to 15th century phials containing her hagiasma, Theodora is rendered with hand gestures typical of saints: holding a cross in front of her breast and raising her other hand in a sign of intercession, with the palm open to the viewer. This iconographical type in all probability depicts the original icon of the 9th century.

A work possibly of the 11 th century, the marble icon T. 148 certainly depicts the Virgin, as is generally accepted. This is indicated by the known iconographical type of the Blachernitissa Orant, from which the hagiasma pours from the holes in both her hands, from the garments typically associated with her with the five-pointed stars at the forehead and the arms, as we find in other icons of her. The maphorion is ornately lined with a fringe on its hem; schematically rendered hanging from the middle of the belt of her dress is her characteristic mandylion, the mappula of a Byzantine lady.

Finally, careful examination of the marble icon showed that the incisions M-P $\Theta Y$, palaeographically consistent with the middle Byzantine period, are contemporary with the icon and worked in the same manner as the relief of the figure. But even were this to have been a later addition, would it not be reasonable to ask whether a later craftsman carving the letters (possibly in Thessaloniki) as well as the person who commissioned the change would have speculated over the figure's identity? It is clear that for them there was no dilemma over "Virgin or Hosia Theodora". The figure was certainly that of the Virgin and her name M-P $\Theta Y$. But, as already noted, the inscription must be contemporary with the figure. 